\title{
Exacerbation of blast-induced ocular trauma by an immune response
}

\author{
Courtney Bricker-Anthony ${ }^{1,2}$, Jessica Hines-Beard ${ }^{1,2}$, Lauren D'Surney ${ }^{3}$ and Tonia S Rex ${ }^{1,2^{*}}$
}

\begin{abstract}
Background: Visual prognosis after an open globe injury is typically worse than after a closed globe injury due, in part, to the immune response that ensues following open globe trauma. There is a need for an animal model of open globe injury in order to investigate mechanisms of vision loss and test potential therapeutics.

Methods: The left eyes of DBA/2 I mice were exposed to an overpressure airwave blast. This strain lacks a fully functional ocular immune privilege, so even though the blast wave does not rupture the globe, immune infiltrate and neuroinflammation occurs as it would in an open globe injury. For the first month after blast wave exposure, the gross pathology, intraocular pressure, visual function, and retinal integrity of the blast-exposed eyes were monitored. Eyes were collected at three, seven, and 28 days to study the histology of the cornea, retina, and optic nerve, and perform immunohistochemical labeling with markers of cell death, oxidative stress, and inflammation.

Results: The overpressure airwave caused anterior injuries including corneal edema, neovascularization, and hyphema. Immune infiltrate was detected throughout the eyes after blast wave exposure. Posterior injuries included occasional retinal detachments and epiretinal membranes, large retinal pigment epithelium vacuoles, regional photoreceptor cell death, and glial reactivity. Optic nerve degeneration was evident at 28 days post-blast wave exposure. The electroretinogram (ERG) showed an early deficit in the a wave that recovered over time. Both visual acuity and the ERG $b$ wave showed an early decrease, then a transient improvement that was followed by further decline at 28 days post-blast wave exposure.

Conclusions: Ocular blast injury in the DBA/2 J mouse recapitulates damage that is characteristic of open globe injuries with the advantage of a physically intact globe that prevents complications from infection. The injury was more severe in DBA/2 J mice than in C57Bl/6 J mice, which have an intact ocular immune privilege. Early injury to the outer retina mostly recovers over time. In contrast, inner retinal dysfunction seems to drive later vision loss.
\end{abstract}

Keywords: Eye trauma, Immune response, Vision loss, Cell death, Neurodegeneration

\section{Background}

Over 186,000 eye injuries were diagnosed in fixed (not deployed) United States military medical facilities between 2000 and 2011 [1]. These injuries were recently projected to cost the United States economy $\$ 25$ billion in healthcare, work lost, and family support. In addition, each year approximately 50,000 United States citizens experience permanent vision loss as a result of trauma [2-4]. While most civilian traumatic eye injuries are

\footnotetext{
*Correspondence: tonia.rex@vanderbilt.edu

'Vanderbilt Eye Institute, Vanderbilt University, 11425 MRB IV, 2213 Garland

Ave., Nashville, TN 37232, USA

${ }^{2}$ Vanderbilt Brain Institute, Vanderbilt University, 11425 MRB IV, 2213 Garland Ave., Nashville, TN 37232, USA

Full list of author information is available at the end of the article
}

unilateral and due to blunt force trauma, a large proportion are bilateral in the military population and are caused primarily by blast wave exposure [5].

Open globe injury refers to an insult that perforates or penetrates the eye, accounting for approximately $40 \%$ of all ocular injuries in military service members $[5,6]$. Visual outcomes are worse after open globe trauma as compared to closed globe due to the increased incidence of ocular inflammation (such as endophthalmitis) that can lead to greater corneal damage, and proliferative vitreoretinopathy [7]. This is in addition to common pathologies shared between open and closed globe trauma, including retinal tears, retinal detachments, choroidal ruptures, and optic nerve atrophy [7].

\section{Ciomed Central}


Treatment for eye trauma has been impeded by the lack of suitable animal models that recapitulate the initial injury. We have developed an experimental system that mimics the primary blast wave experienced by military service members [8]. This system directs a blast of overpressure air at the mouse eye. When directed at the eyes of $\mathrm{C} 57 \mathrm{Bl} / 6 \mathrm{~J}$ mice, the blast induces subtle changes during the first weeks after injury, with significant visual deficits developing over time [9].

In this study we use our eye-directed blast model on a mouse that lacks a molecularly intact blood ocular barrier. The DBA/2 J mouse lacks C5 and CD94, necessary components of the anterior chamber-associated immune deviation (ACAID), which controls ocular immune privilege in the anterior part of the eye [10]. Our goal was to generate a model of open globe trauma by inducing open globe symptoms while retaining a physically intact eye, thus allowing for longitudinal study of injury progression without complications due to infection. We have characterized the effects of blast waves on the cornea, retina, optic nerve, and visual function during the first month post-blast wave exposure.

\section{Methods}

Animals

Three-month-old DBA/2 J ( $\mathrm{n}=85)$, were used in this study (The Jackson Laboratory, Bar Harbor, Maine, United States). Mice were maintained on a 12 hour light/dark cycle and provided access to food and water ad libitum. All experimental procedures were approved by the Institutional Animal Care and Use Committee of Vanderbilt University (protocol \# M/12/132), according to the Association for Assessment and Accreditation of Laboratory Animal Care guidelines. The DBA/2 J mouse is susceptible to developing glaucoma from about six months of age; therefore, all mice were collected at four months of age to avoid glaucoma-related complications [11]. These mice have reactive microglia in the retina at three months of age, indicating a heightened neuroinflammatory state even in the absence of trauma [12]. Therefore, age-matched controls were used throughout the study.

\section{Ocular blast injury}

Blast wave exposure was performed as previously described [8]. Briefly, anesthetized mice were secured and padded within a housing chamber that was slid within a larger tube composed of polyvinylchloride (PVC), which shielded the body and head of the mouse from the blast wave. The outer PVC tube had a machined hole corresponding to the size of a mouse eye. The left eye of the mouse was positioned against a hole in the tube and was aligned with the barrel of the blast device. An overpressure airwave was produced by a modified paintball marker (Empire Paintball, Sewell, New Jersey, United States). Experiments were performed at blast pressures of 23,26 , or 30 psi.

\section{Anterior injuries}

Gross pathology was assessed prior to injury and at three, seven, 14, and 28 days following blast wave exposure using an SZX16 stereomicroscope (Olympus, Center Valley, Pennsylvania, United States). Representative images were taken using a DP71 camera (Olympus, Center Valley, Pennsylvania, United States). Eyes were examined for the presence of corneal abrasions (CA), corneal edema (CE), calcification, corneal growths, blood in the anterior chamber (hyphema), cataracts, corneal neovascularization (CNV), torn irides/pupilomotor deficit (PMD), optic nerve avulsion, lid edema, and torn extraocular muscle tissue.

\section{Ultra-high resolution optical coherence tomography}

The eyes of mice were dilated with $1 \%$ tropicamide. Systane Ultra drops (Alcon, Fort Worth, Texas, United States) were used to keep the eyes moist. The mice were wrapped in gauze, placed in a holding chamber, and head position was stabilized with a bite bar. A Bioptigen ultra-high resolution spectral domain optical coherence tomography (OCT) system with a mouse retinal bore (Bioptigen, North Carolina, United States) was used to image the retinas. Measurements were made using digital calipers in the Bioptigen software.

\section{Visual acuity}

The Optomotry optokinetic nystagmus (OKN) system (Cerebral Mechanics, Lethbridge, Alberta, Canada) was used to assess photopic visual acuity in awake mice $(n=9)$ at three, seven, 14, and 28 days post-blast wave exposure. A step-wise, masked protocol was used. Mice were acclimated to the testing chamber for five minutes prior to the initiation of each test. Spatial frequency for visual acuity was $0.042 \mathrm{c} / \mathrm{d}$.

\section{Flash electroretinogram}

A Diagnosys Espion electrophysiology system (Lowell, Massachusetts, United States) with heated mouse platform was used to perform flash electroretinograms (ERGs) at seven, 14, and 28 days post-blast wave exposure. Dark-adapted mice $(\mathrm{n}=14)$ were anesthetized with a ketamine/xylazine cocktail (Ketaset, Pfizer, New York, New York, United States; AnaSed, Lloyd, Inc., Shanandoah, Iowa, United States) and eyes were dilated with a $1 \%$ tropicamide solution. Mice were exposed to flashes of light ranging from -2 to $2.88 \log \mathrm{cd}^{*} \mathrm{~s} / \mathrm{m}^{2}$ with a flash frequency of $2,000 \mathrm{~Hz}$. For flashes below $-1 \log \mathrm{cd}^{*} \mathrm{~s} /$ $\mathrm{m}^{2}$, the inter sweep delay was 10 seconds, for the $-1 \log$ $\mathrm{cd}^{*} \mathrm{~s} / \mathrm{m}^{2}$ flash it was 15 seconds, and for all remaining flashes the delay was 20 seconds. Oscillatory potentials 
Table 1 Antibodies used in this study

\begin{tabular}{llll}
\hline Antigen & Dilution & Manufacturer & $\begin{array}{l}\text { Catalog } \\
\text { number }\end{array}$ \\
\hline Caspase-1 & $1: 100$ & Millipore, Billerica, MA & AB1871 \\
Caspase-3 & $1: 10$ & Abcam, Cambridge, MA & ab4051 \\
Choline & $1: 25$ & Abcam, Cambridge, MA & ab34419 \\
acetyltransferase & & & \\
Complent 3d & $1: 100$ & Abcam, Cambridge, MA & ab15881 \\
GFAP & $1: 400$ & Dako, Carpinteria, CA & Z0334 \\
Iba1 & $1: 500$ & Wako, Richmond, VA & $019-19741$ \\
Nitrotyrosine & $1: 500$ & Millipore, Billerica, MA & 06-284 \\
RIP1 & $1: 100$ & Santa Cruz, Santa Cruz, CA & sc-7881 \\
RIP3 & $1: 100$ & Santa Cruz, Santa Cruz, CA & sc-47364 \\
\hline
\end{tabular}

GFAP, glial fibrillary acidic protein; Iba1, ionized calcium-binding adapter 1; RIP1, receptor-interacting protein kinase 1; RIP3, receptor-interacting protein kinase 3.

were measured at $3 \log \mathrm{cd} * \mathrm{~s} / \mathrm{m}^{2}$ sampled at $2,000 \mathrm{~Hz}$ with an inter sweep delay of 15 seconds. Amplitudes were measured from baseline to peak.

\section{Tissue collection}

Mice were perfused with $4 \%$ paraformaldehyde (PFA; Electron Microscopy Sciences, Hatfield, Pennsylvania, United States) and phosphate buffered saline (PBS). Following the perfusion, the eyes and optic nerves were placed in either 4\% PFA (for immunohistochemistry) or $4 \%$ PFA with $0.5 \%$ glutaraldehyde (for resin).

\section{Eye histology}

For histological analysis, eyes $(n=20)$ from mice that did not receive eye drops after blast wave exposure were postfixed, bisected, embedded in resin, sectioned on a microtome, and stained with toluidine blue (Fisher, Waltham, Massachusetts, United States). Representative images were collected on an Olympus Provis AX70 (Olympus, Center Valley, Pennsylvania, United States) with a 60x oil objective lens. To quantify retinal pigment epithelium (RPE)

Table 2 Gross pathology after a 23 psi blast wave

\begin{tabular}{llllll}
\hline Type of injury & $\mathbf{0}$ day & $\mathbf{3}$ days & $\mathbf{7}$ days & $\mathbf{1 4}$ days & $\mathbf{2 8}$ days \\
& $\left(\mathbf{1 5 ) ^ { \mathbf { a } }}\right.$ & $\mathbf{1 3}$ & $\mathbf{1 3}$ & $\mathbf{8}$ & $\mathbf{5}$ \\
\hline Corneal abrasion & $2(14)^{\mathrm{b}}$ & 0 & 0 & 0 & $1(20)$ \\
CE & 0 & $9(69)$ & $3(23)$ & $4(50)$ & $5(100)$ \\
CNV & 0 & $4(31)$ & $4(31)$ & $5(63)$ & $4(80)$ \\
Corneal scarring & 0 & $1(8)$ & $3(23)$ & $3(38)$ & $2(40)$ \\
Hyphema & 0 & $1(8)$ & 0 & 0 & 0 \\
Corneal growth & 0 & 0 & $1(8)$ & $1(13)$ & $1(20)$ \\
Torn iris & 0 & 0 & 0 & 0 & 0 \\
Traumatic cataract & 0 & $2(15)$ & $3(23)$ & $3(38)$ & $3(60)$ \\
\hline
\end{tabular}

${ }^{\mathrm{a}}$ Total number of eyes examined. ${ }^{\mathrm{b}}$ Number of eyes with pathology (percentage). CE, corneal edema; CNV, corneal neovascularization.
Table 3 Gross pathology after a 26 psi blast wave

\begin{tabular}{llllll}
\hline Type of injury & $\begin{array}{l}\mathbf{0} \text { day } \\
(\mathbf{2 4})^{\mathbf{a}}\end{array}$ & $\mathbf{3}$ days & $\mathbf{2 3}$ days & $\mathbf{1 4}$ days & $\mathbf{2 8}$ days \\
& $4(17)^{\mathrm{b}}$ & $1(4)$ & $\mathbf{1 4}$ & $\mathbf{9}$ & $\mathbf{7}$ \\
\hline Corneal abrasion & 0 & $16(70)$ & $5(36)$ & $5(56)$ & $6(86)$ \\
CE & $1(4)$ & $4(17)$ & $2(14)$ & $4(44)$ & $2(29)$ \\
CNV & 0 & $2(9)$ & $1(7)$ & $4(44)$ & $5(71)$ \\
Corneal scarring & 0 & $6(26)$ & $1(7)$ & 0 & 0 \\
Hyphema & 0 & 0 & 0 & $1(11)$ & $1(14)$ \\
Corneal growth & 0 & 0 & 0 & $1(11)$ & $2(29)$ \\
Torn iris & 0 & $8(35)$ & $1(7)$ & $1(11)$ & $1(14)$ \\
Traumatic cataract & 0 & & 0 &
\end{tabular}

${ }^{a}$ Total number of eyes examined. ${ }^{b}$ Number of eyes with pathology (percentage). CE, corneal edema; CNV, corneal neovascularization.

damage, a grading scale was developed to classify the vacuoles: 1 (normal, very infrequent and small), 2 (small and infrequent), 3 (small and frequent), 4 (large and infrequent), and 5 (large and frequent). The number of pyknotic nuclei in the outer nuclear layer (ONL) or inner nuclear layer (INL) was quantified within a single section of retina through the middle of each eye.

For immunohistochemistry, eyes $(\mathrm{n}=65)$ were cryoprotected in $30 \%$ sucrose overnight at $4{ }^{\circ} \mathrm{C}$, embedded in Tissue Freezing Medium (Triangle Biomedical, Durham, North Carolina, United States) and then sectioned on a cryostat (Fisher, Pittsburgh, Pennsylvania, United States). Sections of $10-\mu \mathrm{m}$ thickness were collected in round on 12 slides, such that each slide contained representative sections from the entire eye.

\section{Optic nerve histology}

Optic nerves were post-fixed in 1\% osmium tetroxide in $0.1 \mathrm{M}$ cacodylate buffer, dehydrated in a graded ethanol series and embedded in Spurr's resin (Electron Microscopy Sciences, Hatfield, Pennsylvania, United States). Sections of $1-\mu \mathrm{m}$ thickness were collected on a Reichert-Jung Ultracut E microtome (Leica Microsystems, Vienna, Austria) and stained with $1 \%$ p-phenylenediamine in $50 \%$ methanol

Table 4 Gross pathology after a 30 psi blast wave

\begin{tabular}{llllll}
\hline Type of injury & $\begin{array}{l}\mathbf{0} \text { day } \\
(\mathbf{1 6})^{\mathbf{a}}\end{array}$ & $\mathbf{3}$ days & $\mathbf{1 6}$ days & $\mathbf{1 4}$ days & $\mathbf{2 8}$ days \\
\hline Corneal abrasion & $3(19)^{b}$ & $1(6)$ & 0 & $\mathbf{1 1}$ & $\mathbf{1 1}$ \\
CE & 0 & $8(50)$ & $9(64)$ & $10(91)$ & $8(73)$ \\
CNV & 0 & 0 & $4(29)$ & $6(54)$ & $5(45)$ \\
Corneal scarring & 0 & 0 & 0 & $1(9)$ & 0 \\
Hyphema & $1(6)$ & $4(25)$ & $2(14)$ & $2(18)$ & 0 \\
Corneal growth & 0 & 0 & 0 & $3(27)$ & $5(45)$ \\
Torn iris & 0 & 0 & 0 & 0 & 0 \\
Traumatic cataract & 0 & $6(38)$ & $6(43)$ & $6(55)$ & $7(64)$ \\
\hline
\end{tabular}

${ }^{\mathrm{a}}$ Total number of eyes examined. ${ }^{\mathrm{b}} \mathrm{Number}$ of eyes with pathology (percentage). CE, corneal edema; CNV, corneal neovascularization. 
(Sigma-Aldrich, St Louis, Missouri, United States). Optic nerve sections were examined for the presence of degenerating axons on an Olympus Provis AX70 microscope using a 100x oil immersion objective lens.

\section{Retina immunohistochemistry}

Slides were rinsed with PBS (Sodium chloride [SX0420-3, Millipore, Darmstadt, Germany], potassium chloride [P39 11-500, Sigma-Aldrich, St. Louis, Missouri], sodium phosphate dibasic [S374-500, Fisher, Pittsburgh, Pennsylvania, United States] and potassium phosphate monobasic [P285500, Fisher, Pittsburgh, Pennsylvania, United States]) and incubated at room temperature in normal donkey serum (530-100, Millipore) at 1:20 in 0.1 M phosphate buffer (Sodium phosphate monobasic [BP329-500, Fisher, Pittsburgh, Pennsylvania, United States] and sodium phosphate dibasic [S374-500, Fisher, Pittsburgh, Pennsylvania, United States]) with $0.5 \%$ bovine serum albumin (BP1600-100, Fisher, Pittsburgh, Pennsylvania, United States) and 0.1\% Triton X 100 (H5142, Promega, Madison, Wisconsin, United States) (PBT) for two hours. The slides were incubated overnight at $4^{\circ} \mathrm{C}$ in primary antibody in PBT (Table 1), rinsed with PBS and incubated with a secondary antibody (Life Technologies, Grand Island, New York, United States) for two hours at room temperature. Slides were rinsed with PBS and mounted in Vectashield Mounting medium with 4,6-diamidino-2-phenylindole (DAPI; Vector Laboratories, Burlingame, California, United States) for imaging on a Nikon Eclipse epifluorescence microscope (Nikon, Melville, New York, United States) or Olympus FV-1000 confocal microscope (Olympus, Center Valley, Pennsylvania, United States). Imaging on the Olympus FV-1000 microscope was performed through use of the Vanderbilt University Medical Center Cell Imaging Shared Resource.

\section{Tdt dUTP nick end labeling quantification}

Eye sections from mice treated with non-medicated eye drops were labeled with the Tdt dUTP nick end labeling
(TUNEL) Apoptosis Detection Kit adhering to the manufacturer's protocol (Merck Millipore, Darmstadt, Germany) and mounted with Vectashield Mounting Medium with DAPI. TUNEL-positive cells within the ONL, INL and ganglion cell layer (GCL) were counted and the lengths of the regions with TUNEL-positive cells (affected regions) were measured using NIS Elements Advanced Research software (Nikon, Melville, New York, United States). The total length of each retinal section with TUNEL-positive cells (affected section) was also measured. In order to determine the percentage of the retina with cell death, we measured and summed the lengths of all sections on the slide. Then, we divided the sum of affected region lengths by the total length of all sections and multiplied this value by 100 , which yielded the percentage of retina with TUNELpositive cells.

\section{Statistical analysis}

All statistical analyses were calculated using Graphpad Prism software (San Diego, California, United States). A one-way ANOVA with a Bonferroni post-hoc test was used to analyze ERG and visual acuity data. The means \pm SEM were calculated and presented for each data set.

\section{Results}

\section{Ocular trauma induces corneal and lens damage}

Blast wave exposure caused numerous anterior injuries that, in some cases, varied depending on time after the blast (Tables 2, 3, and 4). This is in contrast to the lack of anterior pathologies in the majority of $\mathrm{C} 57 \mathrm{Bl} / 6$ mice after eye blast $[8,9]$. In both cases, no eye drops or ointments were provided in order to detect all pathologies caused by an eye-directed blast to the naïve eye. Representative images of these pathologies after exposure to a 26 psi blast wave are shown in Figure 1. The eyes appeared normal immediately after the blast wave, but at three days significant pathologies were present including

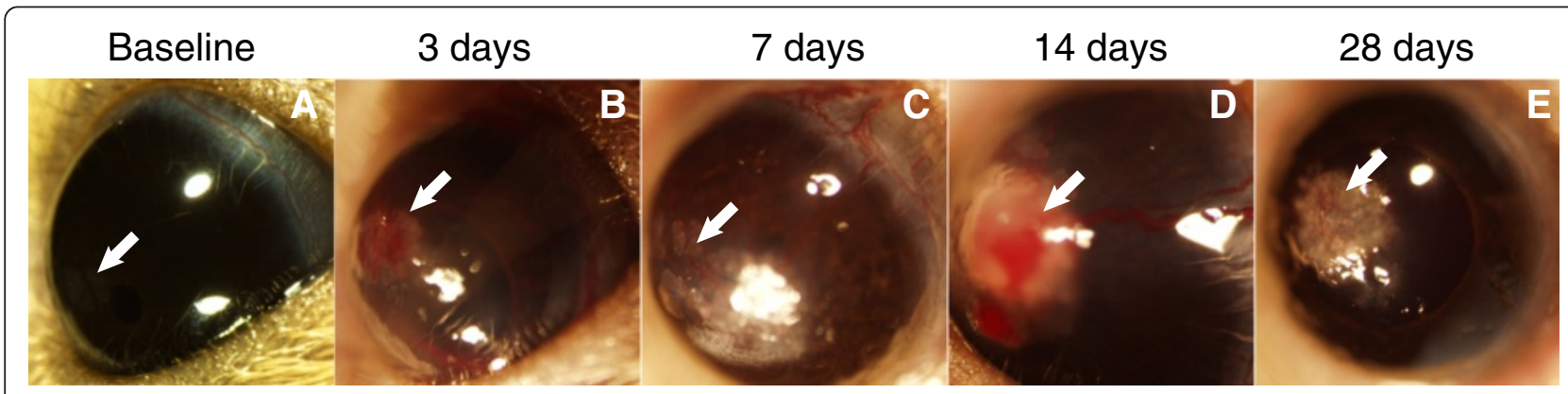

Figure 1 Blast trauma injures the ocular surface. (A) The majority of mice had calcium deposits in the cornea at baseline. Images showing the most common anterior pathologies detected at three, seven, 14, and 28 days post-blast wave exposure. (B) Corneal edema and hyphema at three days post-blast wave exposure. (C) Corneal edema and neovascularization at seven days post-blast wave exposure. (D) A corneal growth with neovascularization and hyphema at 14 days post-blast wave exposure. (E) Corneal scarring and neovascularization at 28 days post-blast wave exposure. Arrows indicate pathologies. 
Figure 2 Corneal damage persists out to 28 days post-blast wave exposure. (A-C) Brightfield micrographs of cornea histology. (A) In control corneas the corneal epithelium (epi), stroma, and endothelium (endo) are well organized and show no signs of pathology. (B) At three days post-blast wave exposure, the epi is disorganized and the stroma contains neutrophils. (C) At 28 days post-blast wave exposure the epi is thin and blood vessels are evident in the stroma. The scale bar in (A) is $50 \mu \mathrm{m}$ and also applies to (B) and (C).

$\mathrm{CE}$, hyphema, cataracts, and a few cases of CNV. The incidence of $\mathrm{CE}$ after a 23, 26, or 30 psi blast wave remained high up to 28 days; $100 \%, 86 \%$, and $60 \%$, respectively. The incidence of hyphema in all blast groups peaked at three days after blast wave exposure and was completely absent at 28 days post-blast wave exposure. The percentage of eyes with hyphema at three days was $8 \%, 26 \%$, and $25 \%$ after a 23,26 , and 30 psi blast wave, respectively. In contrast, the number of eyes with $\mathrm{CNV}$ increased over time post-blast wave exposure. At three days post-blast wave exposure $31 \%, 17 \%$, and $0 \%$ of 23 , 26 , and 30 psi eyes, respectively, exhibited signs of CNV. At 28 days $80 \%, 29 \%$, and $45 \%$ of 23,26 , and 30 psi eyes, respectively, had CNV. Exposure to a 26 psi blast wave induced the most reproducible and clinically relevant damage profile. Therefore, this pressure level was used for the remaining experiments.

Consistent with the gross pathology observations, corneal damage was apparent by histology following blast wave exposure (Figure 2). Disruption of the epithelium (epi), stromal edema, and peripheral immune infiltrate were present at three days post-injury (Figure 2B). In all 28-day post-injury corneas, the epi was thin and disorganized and the stroma contained neovascularization and pockets of blood (Figure 2C).

The high frequency of corneal opacities after blast wave exposure excluded the majority of eyes from functional assessments. We hypothesized that the CE and CNV developed secondarily due to dry eye. To test this, mice were treated once with non-medicated, viscous eye drops immediately post-blast wave exposure. This prevented $90 \%$ of the corneal damage, including CE. Therefore, to perform functional (OKN, ERG) and in vivo anatomical (OCT) assessments, all additional mice received eye drops.

Corneal cell death was examined in mice that received non-medicated eye drops. In the control cornea, occasional TUNEL-positive cells were detected in the epi as a result of normal cellular turnover (Figure 3A). At three days postinjury, TUNEL-positive cells were increased in the epi and were detected in the stroma (Figure 3C). There were no changes in immunolabeling for receptor interacting proteins 1 and 3 (RIP1, RIP3; markers of necroptosis) at three days when compared to control (Figure 3D). At 28 days post-injury, TUNEL-positive cells (Figure 3E), 


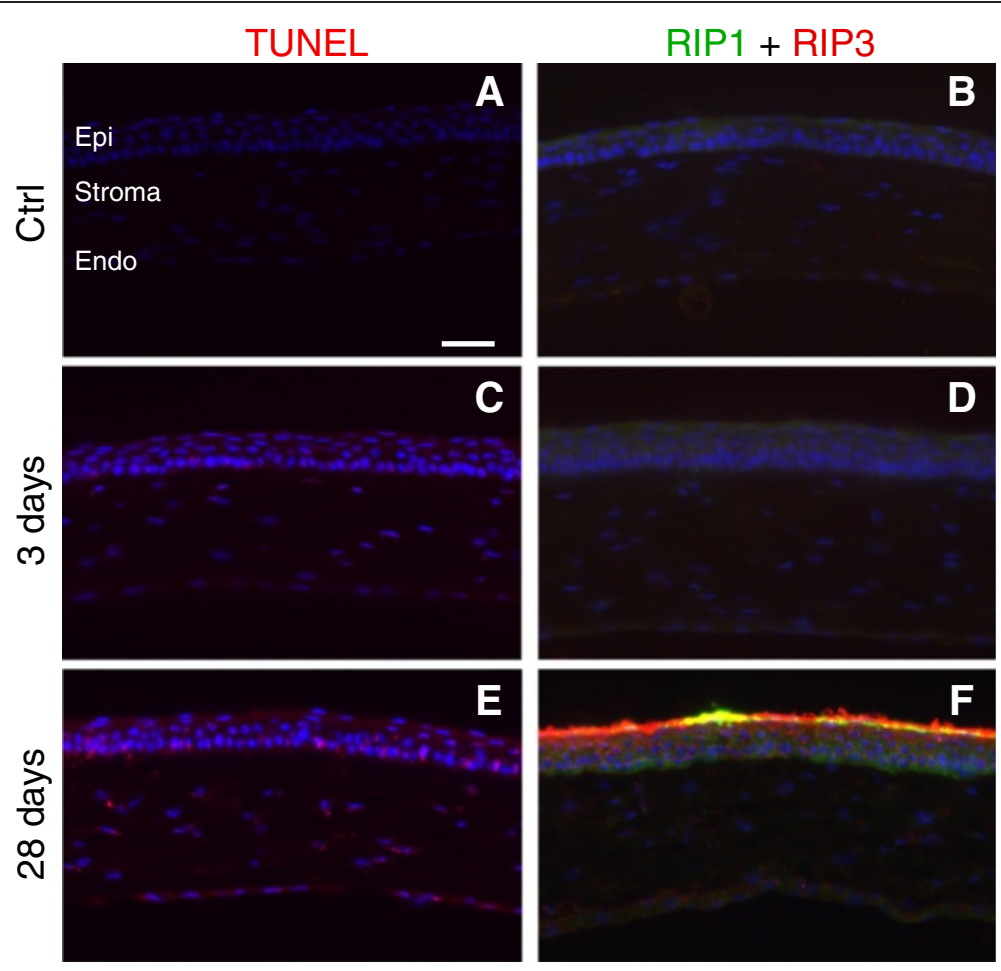

Figure 3 Cell death affects each layer of the cornea and is necroptotic. (A, C, E) Epifluorescence micrographs of TUNEL (red) and DAPI (blue). (B, D, F) Epifluorescence micrographs of RIP1 (green), RIP3 (red), and DAPI (blue). (A) In control corneas, TUNEL is rare and restricted to the epithelium (epi). (B) The control cornea is negative for both RIP1 and RIP3. (C) At three days post-blast wave exposure TUNEL is increased in the epi and is present in the stroma. (D) The cornea is still negative for RIP1 and RIP3 at three days post-injury. (E) At 28 days post-blast wave exposure, all three layers of the cornea are positive for TUNEL. (F) Both RIP1 and RIP3 are present in the epi, as well some light labeling in the endothelium (endo) and stroma. The scale bar in (A) is $50 \mu \mathrm{m}$ and applies to all of the images in Figure 3.
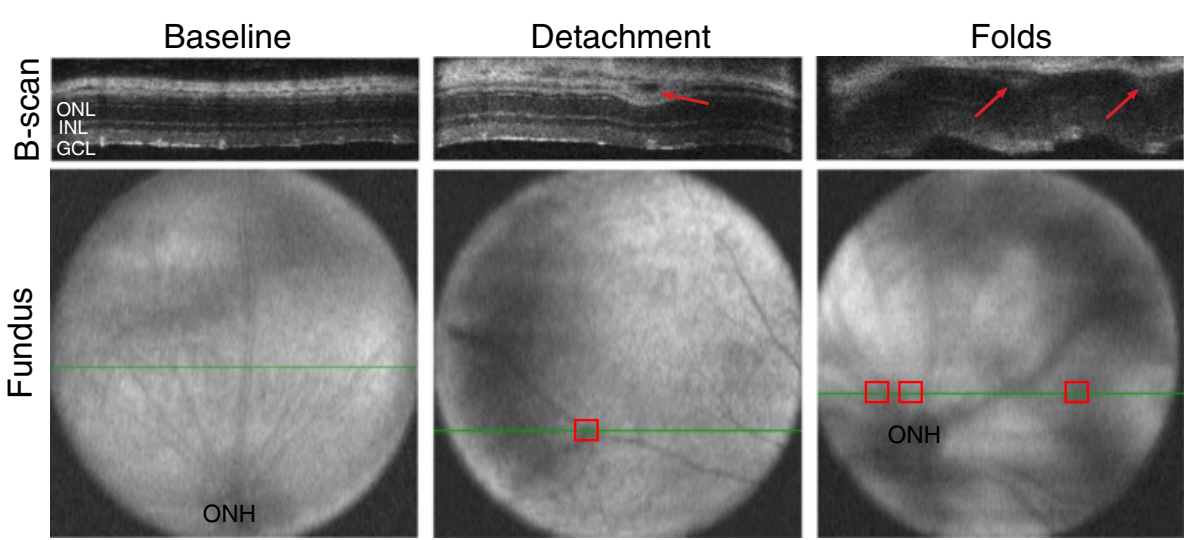

Figure 4 Blast wave exposure damages the neural retina. At baseline, each layer of the retina appears normal in the b-scan image and the corresponding fundus image shows no signs of pathology ( $\mathrm{ONH}$ : optic nerve head). The green lines in the fundus images denote the location of the b-line scan images. An example of a retinal detachment after blast wave exposure is shown. It appears as a dark area between the retinal pigment epithelium (RPE) and photoreceptors (arrow) and as a dark shadow in the mid-peripheral region of the retina fundus image (box). An example of retinal folds with corresponding retinal detachments (arrows; boxes) is shown. ONL: outer nuclear layer, INL: inner nuclear layer, GCL: ganglion cell layer. 


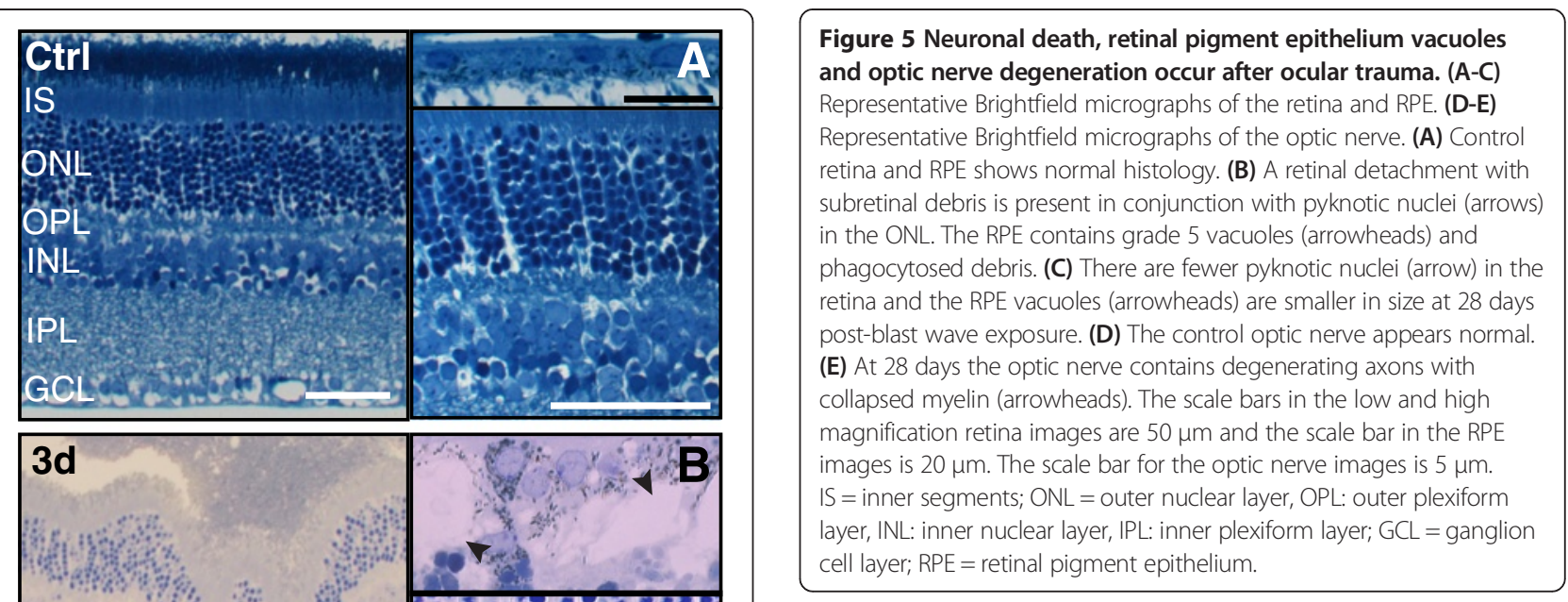

and increased RIP1 and RIP3 immunolabeling were detected in all layers of the cornea (Figure 3F).

\section{Ocular trauma causes retinal detachments}

The majority of retinas exposed to a blast wave appeared normal at all time points by OCT imaging, despite repositioning the eye multiple times during imaging to examine all retinal quadrants (Figure 4). When retinal detachments were detected they were primarily in the mid-peripheral retina and less frequently near the optic nerve head $(\mathrm{ONH})$. At three days post-blast wave exposure $(n=5), 40 \%$ of eyes had a single retinal detachment that had an average height of $0.03 \mathrm{~mm} \pm 0.005$ (Figure 4). At seven days post-blast wave exposure $(\mathrm{n}=10)$, only one eye had retinal detachments. The average height and number of the detachments at seven days was $0.05 \mathrm{~mm} \pm 0.03$ and six per eye, respectively. One retina had a wavy appearance suggestive of epiretinal membranes and multiple retinal detachments (Figure 4). No detachments were observed at 14 days post-injury $(n=6)$, but it is possible that detachments were missed during imaging. At 28 days $(\mathrm{n}=10)$, only the retina that appeared wavy at the seven-day time point had retinal detachments, a total of two, averaging $0.03 \mathrm{~mm} \pm 0.00$ in height.

\section{Blast wave exposure damages the neural retina, retinal} pigment epithelium, and optic nerve

In the normal RPE, there were no vacuoles or debris accumulation present (Figure 5A). In contrast, in mice that did not receive eye drops, the RPE contained grade 5 vacuoles at three days post-blast wave exposure in the majority of eyes $(67 \%, n=3$, Figure $5 B)$. At 28 days after blast wave exposure, the RPE vacuoles had decreased in size to grade $2(\mathrm{n}=7$, Figure $5 \mathrm{C})$. At both time points the RPE vacuoles were present throughout the retina. Subretinal debris, consisting of red blood cells and photoreceptor inner and outer segments, were detected in areas of retinal detachments at three days post-blast 

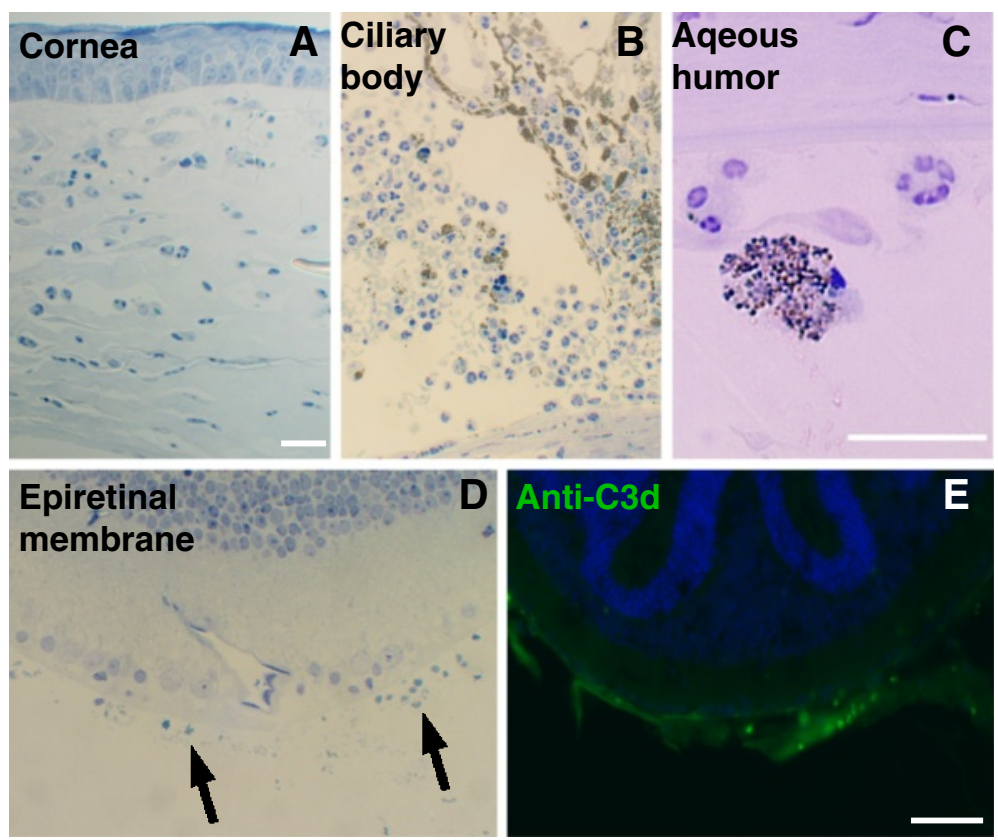

Figure $\mathbf{6}$ Immune infiltrate appears after blast wave exposure. (A-C) Immune infiltrate is present in the anterior chamber, including the cornea (A) and the aqueous humor near the ciliary body (B) and the aqueous humor near the cornea (C). (D) An epiretinal membrane after blast wave exposure (arrows). (E) The epiretinal membranes are positive for complement $3 \mathrm{~d}$ (C3d) indicating that they are immune-mediated. The scale bar in (A) is $50 \mu \mathrm{m}$ and also applies to (B) and (D); the scale bar in (C) is $5 \mu \mathrm{m}$; the scale bar in (E) is $25 \mu \mathrm{m}$.

wave exposure (Figure 5B), but appeared to resolve at 28 days post-blast wave exposure (Figure $5 \mathrm{C}$ ). RPE damage was less severe and more focal, with most of the RPE appearing normal, when mice were given nonmedicated eye drops immediately after blast (Additional file 1: Figure S1).

While much of the post-blast wave retina looked normal, clusters of pyknotic nuclei were observed at three and 28 days (Figure 5B, C). The average number of pyknotic nuclei at three days post-blast wave exposure was $14 \pm 10$ in the ONL and $31 \pm 21$ in the INL. At 28 days post-blast wave exposure, the average number of pyknotic nuclei decreased to $3 \pm 2$ and $0 \pm 0$ in the ONL and INL, respectively. Optic nerves from the first week postinjury (data not shown) looked the same as those from controls (Figure 5D). In contrast, degenerating axons with collapsed myelin were prevalent at 28 days postinjury (Figure 5E).

In eyes that did not receive eye drops, immune infiltrate was present in a subset of eyes (33\%) at three days post-blast wave exposure. Infiltrate was detected in both the anterior and posterior portions of the eye, including the cornea, aqueous humor, vitreous humor, and surface of the retina (Figure 6A-C). Immune-mediated (complement 3d, C3d-positive) epiretinal membranes were occasionally detected in areas of retinal detachment (Figure 6D-E). In eyes that received non-medicated eye drops, no immune cells were detected in the anterior half of the eye, only macrophages were present in the posterior eye and only in the subretinal space, and no epiretinal membranes were detected (Additional file 2: Figure S2).

\section{Regional cell death occurs at multiple time points post- blast wave exposure}

After blast wave exposure, all retinas had areas with TUNEL-positive cells (affected areas) at three $(\mathrm{n}=5)$ and seven days post-injury $(\mathrm{n}=9)$, while $82 \%$ of retinas were TUNEL-positive at 28 days post-injury $(\mathrm{n}=11)$. Cell death was typically present in patches and not evenly distributed across the retina (Figure 7). These affected areas were primarily in the mid-peripheral retina, but occasionally were also detected in central retina (Figure 7A). Representative images of TUNEL in affected and unaffected areas of retina three days after blast wave exposure are shown in Figure 7D, E. Areas with retinal folds had the highest density of TUNEL-positive cells.

The percentage of total retina containing TUNELpositive cells, density of TUNEL-positive cells, and retinal layer affected were quantified (Figure 8). The majority of TUNEL-positive nuclei, $82 \%$, were located in the ONL at three days after injury (Figure 8A). A smaller percentage of TUNEL-positive cells were detected in the INL and GCL, $12 \%$ and $6 \%$, respectively, at three days post-blast wave exposure (Figure 8A). This ratio was similar at seven and 28 days post-blast wave exposure: $70 \%$ and $83 \%$, 


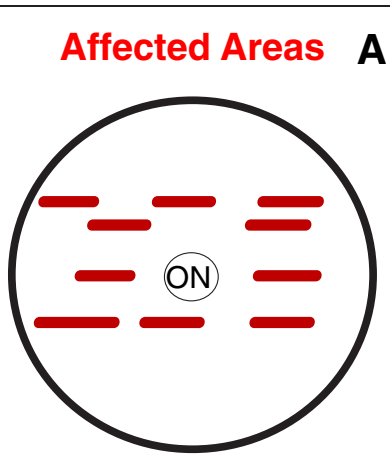

Ctrl

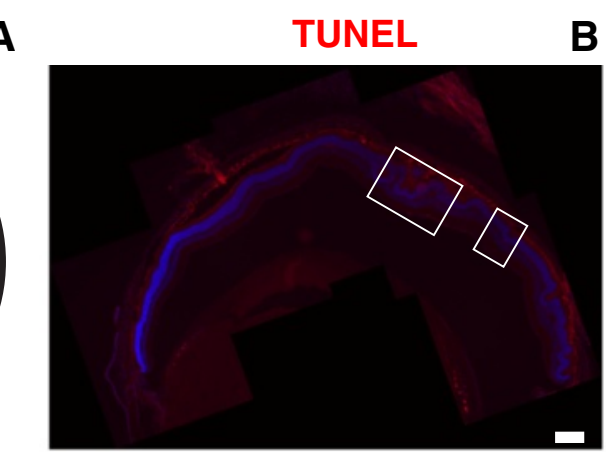

Affected

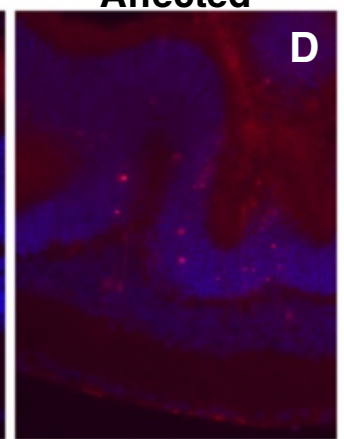

Unaffected

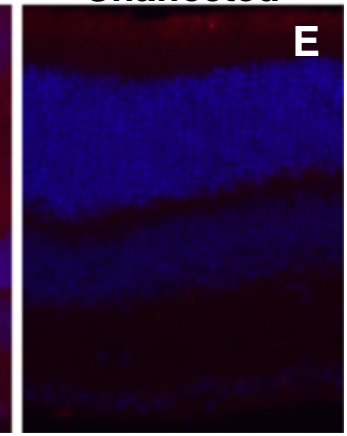

Figure $\mathbf{7}$ Cell death occurs in small, focal areas after blast wave exposure. (A) A schematic representing an enface view of the retina showing the average number and distribution of affected (TUNEL-positive) areas (red bars) detected in retinal cross-sections collected in serial through the eye three days post-blast wave exposure. (B) Montage of low magnification epifluorescence micrographs of a retina three days post-blast wave exposure. White boxes indicate affected areas (scale bar is $250 \mu \mathrm{m}$ ). (C-E) Higher magnification epifluorescence micrographs of TUNEL (red) and DAPI (blue) in a control retina (C), and affected (D) and unaffected (E) regions of the retina shown in (B). The scale bar in (C) represents $50 \mu \mathrm{m}$ and also applies to (D) and (E). ON = optic nerve head; $\mathrm{GCL}=$ ganglion cell layer; INL = inner nuclear layer; ONL = outer nuclear layer.

respectively, located in the ONL; $27 \%$ and $16 \%$, respectively, detected in the INL; and $3 \%$ and $1 \%$, respectively, located in the GCL. TUNEL-positive nuclei were present in $13 \pm 8 \%$ of the retina at three days post-injury, $2 \pm 0.2 \%$ at seven days post-blast wave exposure, and $5 \pm 1 \%$ of the retina at 28 days post-blast wave exposure (Figure $8 \mathrm{~B}$ ). When calculated in terms of total retina length, the density of TUNEL was very low, but retained the same trend of higher levels at three days as compared to 28 days. The number of TUNEL-positive nuclei per $\mathrm{mm}$ total retina was $15 \pm 9,0.1 \pm 0.1$, and $11 \pm 4$ at three, seven, and 28 days post-blast wave exposure (Figure $8 \mathrm{C}$ ). Within the affected regions, the density of TUNEL-positive cells was $87 \pm 44$, $10 \pm 3$, and $215 \pm 57$ nuclei per mm retina at three, seven, and 28 days after blast wave exposure, respectively (Figure 8D). These results demonstrate that the area occupied by TUNEL-positive cells decreases over time, but the density of TUNEL-positive cells within affected areas increases.

\section{Cell death pathway markers increased after blast wave exposure}

Since TUNEL is a method for detecting dying cells in general (it is not specific for any cell death pathway
[13]), we next used markers of apoptosis, necroptosis, and pyroptosis to gain insight into how the cells were dying. Very few caspase-3-positive nuclei were detected in the ONL at three, seven, and 28 days postinjury, even within areas of extensive cell death (data not shown). Caspase-1, a marker for pyroptosis (inflammation-mediated cell death), was present within the INL and GCL throughout the control retinas (Figure 9D). At three days post-injury $(n=3)$, only one third of retinas exhibited caspase-1-positive cells in the INL and GCL (Figure 9E). At 28 days after blast wave exposure $(n=5)$, all retinas were caspase-1negative (Figure 9F). In the $\mathrm{C} 57 \mathrm{Bl} / 6$ mouse caspase- 1 labeling in ChAT-positive cells increased over time post-blast wave exposure [9]. Immunolabeling with anti-ChAT in DBA/2 J mice showed that these cells were still present even at 28 days post-blast wave exposure (data not shown).

In contrast, labeling for markers for necroptosis (programmed necrosis; RIP1 and RIP3) was increased after blast wave exposure. In the normal retina, RIP1 localized to the Müller glia, the inner plexiform layer (IPL), and the INL, with some light staining in the outer plexiform layer (OPL; Figure 9A). Light RIP3 

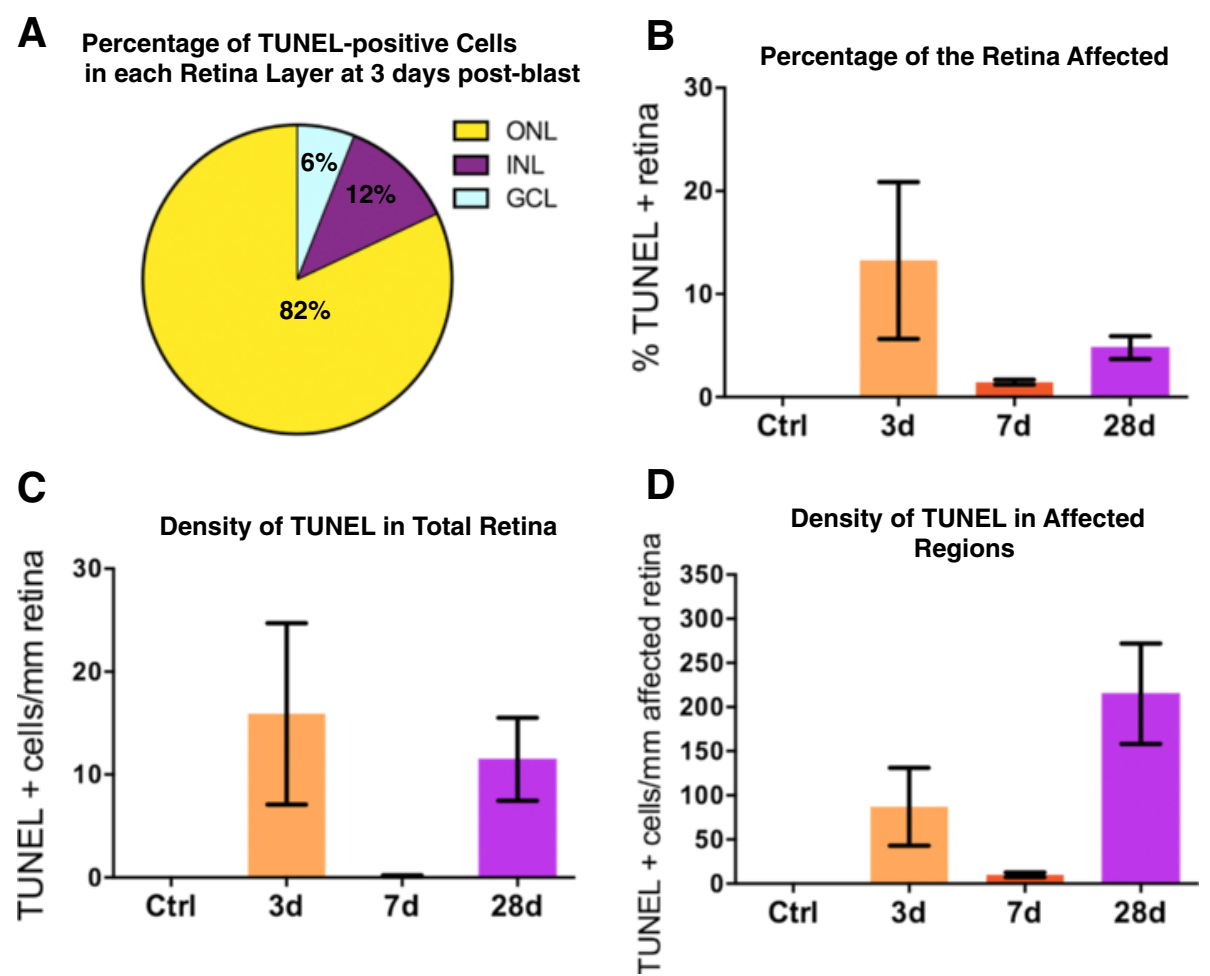

Figure 8 Cell death occurs in two waves after blast wave exposure. (A) Pie chart showing the distribution of TUNEL-positive cells through the retinal layers after blast wave exposure. (B) The percentage of total retina containing TUNEL-positive cells at each time point. (C) The average number of TUNEL-positive cells per mm total retina after blast wave exposure. (D) The average number of TUNEL-positive cells per mm within the affected areas after blast wave exposure. Error bars represent SEM for each time point. GCL = ganglion cell layer; INL = inner nuclear layer; $\mathrm{ONL}=$ outer nuclear layer.

staining in the normal retina was restricted to the GCL, IPL, and INL (Figure 9A). At three days postinjury $(\mathrm{n}=4)$, RIP1 increased in the ONL, INL, and Müller glia, while RIP3 increased in the ONL, INL, IPL, and GCL (Figure 9B). As cell death progressed at 28 days post-blast wave exposure $(\mathrm{n}=5)$, RIP1 remained elevated in the ONL and INL, while RIP3 remained elevated in the IPL and maintained some light labeling in the ONL (Figure 9C).

\section{Protein nitration increases in the retina after blast wave} exposure

In the control retina, nitrotyrosine immunolabeling was light and restricted to the inner retina (Figure 10A). Three days after blast wave exposure $(n=5)$, immunolabeling was greatly increased throughout both the inner and outer retina (Figure 10B). In eyes that received eye drops this increase was limited to focal areas (Additional file 3: Figure S3). The immunolabeling seemed less increased, but was still elevated in both the inner and outer retina at 28 days post-blast wave exposure and spread across the entire retina in eyes that received eye drops (Figure 10C, $\mathrm{n}=5$ ).

\section{Glial reactivity increases in the retina after blast wave exposure}

In the normal retina, glial fibrillary acidic protein (GFAP) immunolabeling was restricted to the Müller glia end-feet and astrocytes (Figure 11A). At both three $(n=5)$ and 28 days ( $\mathrm{n}=9$ ) post-injury, GFAP immunolabeling was increased in the Müller cell processes (Figure 11B, C). In age-matched control retinas, immunolabeling with ionized calcium binding adaptor molecule 1 (IBA-1) showed that microglia were restricted to the inner retina and were fairly low in density (Figure 11D). Microglia were more prevalent after blast wave exposure when compared to controls beginning at three days $(n=5)$ post-blast wave exposure (Figure 11E-F). Reactive microglia, which are amoeboid in appearance, were detected (Figure 11E-F, inserts). GFAP immunolabeling was the same in all eyes regardless of treatment or lack thereof with eye drops. However, there was less of an increase in reactive microglia in eyes that received eye drops after blast (Additional file 4: Figure S4).

\section{Ocular trauma results in visual deficits}

The ERG a wave amplitude $\left(\mathrm{a}_{\max }\right)$, a measure of photoreceptor function, was diminished to approximately $40 \%$ 


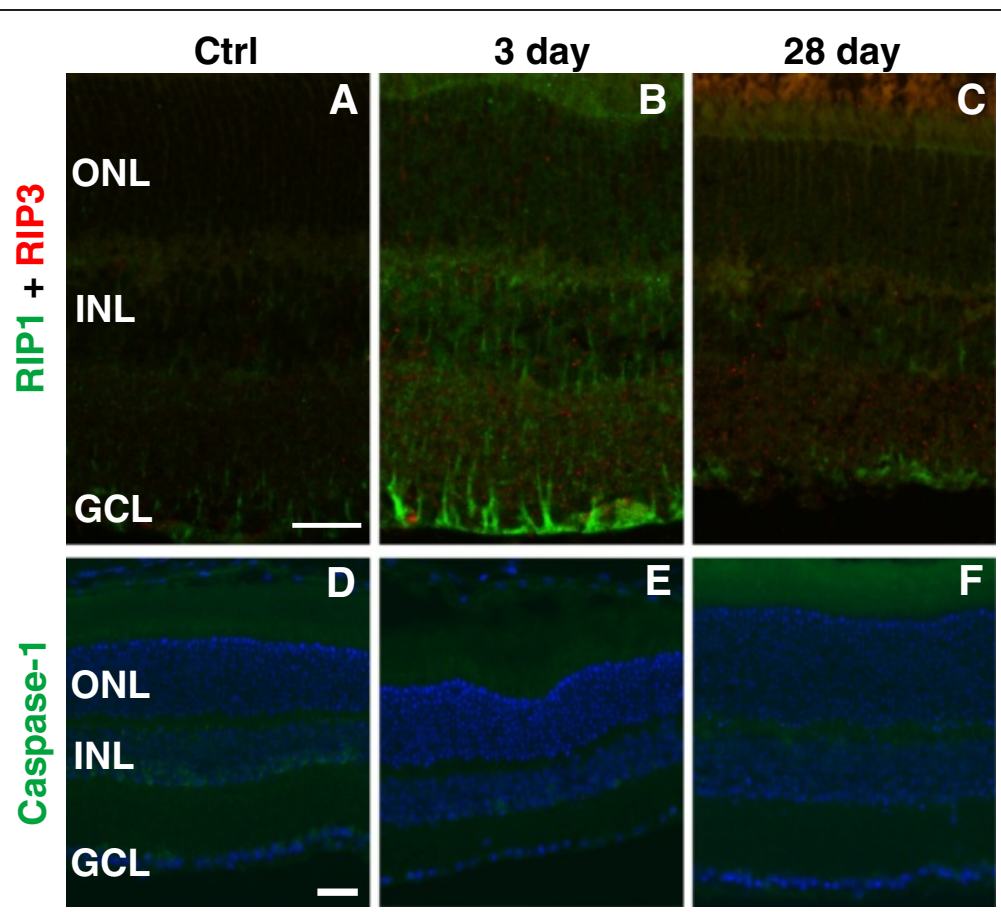

Figure 9 Changes in cell death markers after blast wave exposure suggest a non-apoptotic mode of cell death. (A-C) Confocal micrographs of retinas immunolabeled for RIP1 (green) and RIP3 (red; scale bar is $30 \mu \mathrm{m}$ ). (D-F) Epifluorescence micrographs of Caspase-1 (green) immunolabeling and DAPI (blue; scale bar is $25 \mu \mathrm{m}$ ). GCL = ganglion cell layer; INL = inner nuclear layer; ONL = outer nuclear layer.

of baseline at seven days post-blast wave exposure based on an average of the responses at each light intensity (Figure 12A). At 14 days post-blast wave exposure, a statistically significant decrease is still present, but the level of decrease is less substantial at an average of $55 \%$ of baseline. At 28 days post-blast wave exposure, the $\mathrm{a}_{\max }$ was similar to baseline at the brightest flash, but was still reduced at the other flash intensities (Figure 12A). The average deficit from baseline at the lower light intensities was $63 \%$ at 28 days post-blast wave exposure. The ERG $b$ wave amplitude $\left(b_{\max }\right)$, a measure of inner retinal function, was similar to baseline at the brightest light intensity at all time points post-blast wave exposure (Figure 12B). However, at all other light intensities, there was a deficit averaging $52 \%$ below baseline at seven days post-blast wave exposure, $74 \%$ of baseline at 14 days post-blast wave exposure (note: not statistically significant), and $48 \%$ of baseline at 28 days post-blast wave exposure. The decrease in the $b_{\max }$ at seven days postblast wave exposure seems to correspond to the decrease in the $\mathrm{a}_{\max }$ at this time point (Figure 12B). In contrast, the decrease in the $b_{\max }$, particularly at 0 and $1 \log \mathrm{cd}^{*} \mathrm{~s} /$ $\mathrm{m}^{2}(P<0.01)$ at 28 days post-blast wave exposure was greater than at earlier time points despite a recovery of $a$ wave. The oscillatory potentials (OP), a measure of amacrine and interneuron cell function, were also affected after blast wave exposure (Figure 12C). The OP1 was diminished at all time points after blast wave exposure $(P<0.01)$. OP2 was significantly lower than baseline at both seven and 28 days $(P<0.01)$. OP3 appeared lower than baseline, but only reached statistical significance at seven days post-blast wave exposure $(P<0.01)$. A significant decline in visual acuity was observed at three $(0.40 \pm 0.06, P<0.01), 14(0.25 \pm 0.07$, $P<0.01)$, and $28(0.07 \pm 0.03, P<0.01)$ days post-blast wave exposure (Figure 12D).

\section{Discussion}

The majority of the anterior ocular pathologies appear to be secondary to $\mathrm{CE}$ and dry eye (for example, CNV) as they were almost completely eliminated by treatment with lubricating, non-medicated eye drops given once, acutely, after blast wave exposure. The blast wave may disrupt the tear film, which we artificially restored with the eye drops. A recent study reported tear film deficiencies in blast-exposed veterans [14]. Preserving the corneal fluid barrier limited immune infiltrate, oxidative stress, and microglial reactivity after blast, but had no effect on macroglial reactivity, extent of cell death, or optic nerve degeneration.

A large influx of immune cells into the eye occurred after blast wave exposure in DBA/2 J mice but not in C57Bl/6 mice, which have a fully intact ACAID. The immune response after trauma elicited formation of epiretinal membranes, increased nitrosative stress, greater cell death, and more severe vision loss than was detected in 


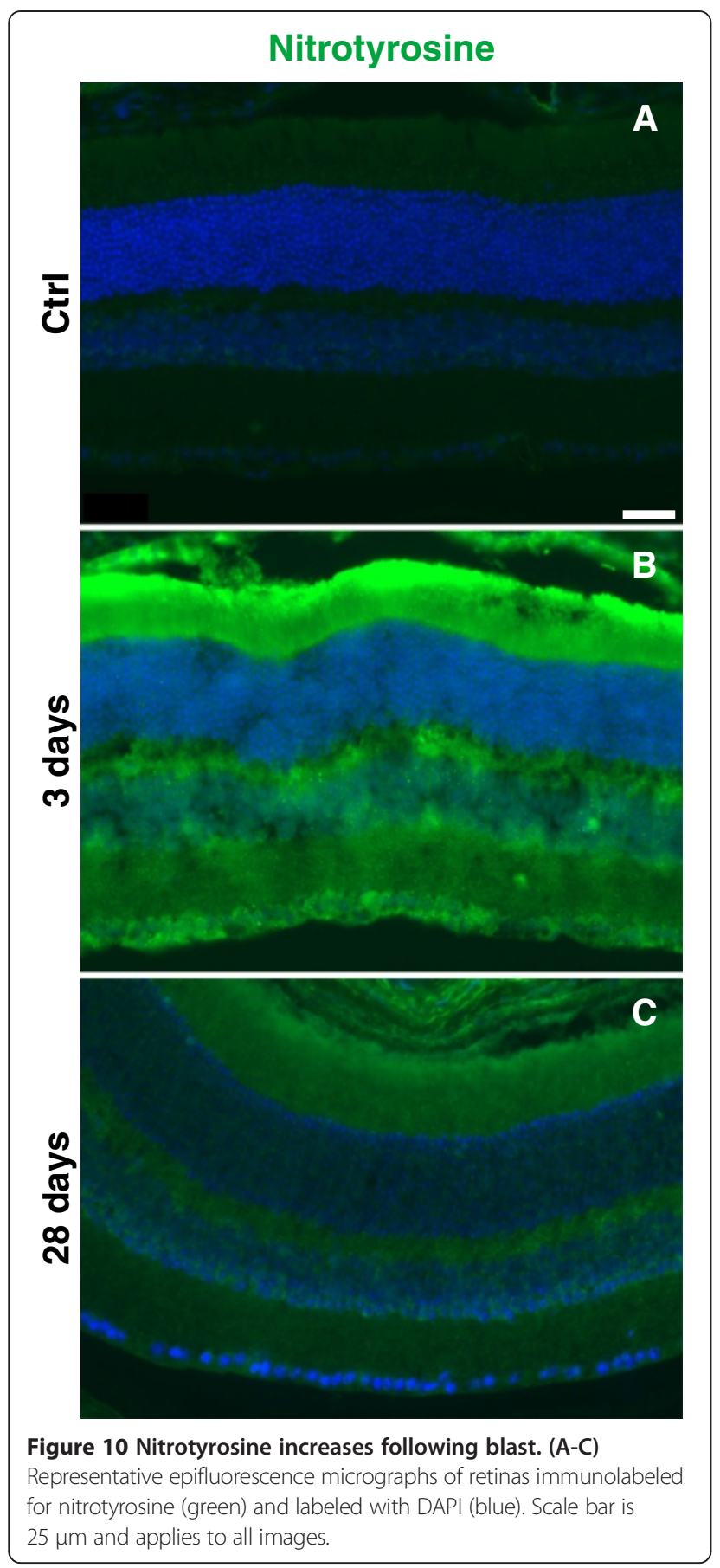

the $\mathrm{C} 57 \mathrm{Bl} / 6$ mouse [9]. Another major difference between these two strains was the acute damage to the RPE after blast wave exposure in DBA/2 J mice. Remarkably, despite the early and dramatic vacuolization and significant amount of subretinal debris present within days after blast wave exposure, the RPE and subretinal space appeared near normal at 28 days after injury. This shows the resiliency of these cells and their large capacity to phagocytose and remove debris. The differences in response to blast wave exposure by these two strains of mice is consistent with comparisons between closed globe and open globe trauma patients [7].

As in the retinas of $\mathrm{C} 57 \mathrm{Bl} / 6$ mice post-blast wave exposure, the cell death pathway appears to be nonapoptotic in DBA/2 J mice [9]. In both strains of mice we detected increases in markers for necroptosis after blast wave exposure, suggesting that necroptosis is the main cell death pathway activated. The peak of cell death is earlier in DBA/2 J mice; three days as compared to 28 days in $\mathrm{C} 57 \mathrm{Bl} / 6$ mice [9]. Also, more retinal ganglion cells (RGC) were TUNEL-positive in DBA/2 J mice and damage to the optic nerve was more substantial. Unlike the whole body/head blast models $[15,16]$, photoreceptor cell death was present in this model and in a trinitrotoluene (TNT) blast study [17]. The discrepancies may be due to the physics of head movement in their model as compared to the restrained head in our model and that of Zou et al. [17]. Similar to Mohan et al., we detect RGC death and degeneration in the optic nerve [16]. They reported axon degeneration and RGC loss at 10 months post-blast wave exposure, while we show that it may begin as early as one month.

Our blast pressure is comparable to the low-level blast group exposed to TNT by Zou et al. [17]. Despite this, the histology and labeling for nitrosative damage and GFAP in this study is more comparable to their findings in the high-level blast exposure group at two weeks post-injury [17]. We suspect that these differences are due to the increased damage caused by immune infiltrate as blast wave-exposed $\mathrm{C} 57 \mathrm{Bl} / 6$ mice show a phenotype more similar to their low-level blast group, as would be expected. In fact there is also evidence of immune infiltrate in their high-level blast group, but not in their low-level blast group retinas [17].

One of the most surprising results was the biphasic decrease in the flash ERG. Acutely post-blast wave exposure, the deficits in visual acuity and in the ERG amplitude appear to be driven by damage to the outer retina. The ERG amplitude decrease was photoreceptor-driven since the decrease in the $a_{\max }$ was greater than that for the $b_{\max }$. These deficits in visual function correlated with the presence of retinal detachments and extensive vacuolization and damage to the RPE in the first week after injury. The recovery of the OKN at seven days post-blast wave exposure may reflect partial resolution of the retinal detachments in combination with an improvement in the OKN response as a result of repeat testing $[9,18]$. A similar dip and recovery of visual function was reported by us, and others, after blast wave exposure $[9,16]$, and is also seen in patients whose visual acuity can drop after trauma, but recover over time $[5,19]$.

At 28 days post-blast wave exposure the profile is very different. The ERG $a_{\max }$ is improved as compared to 

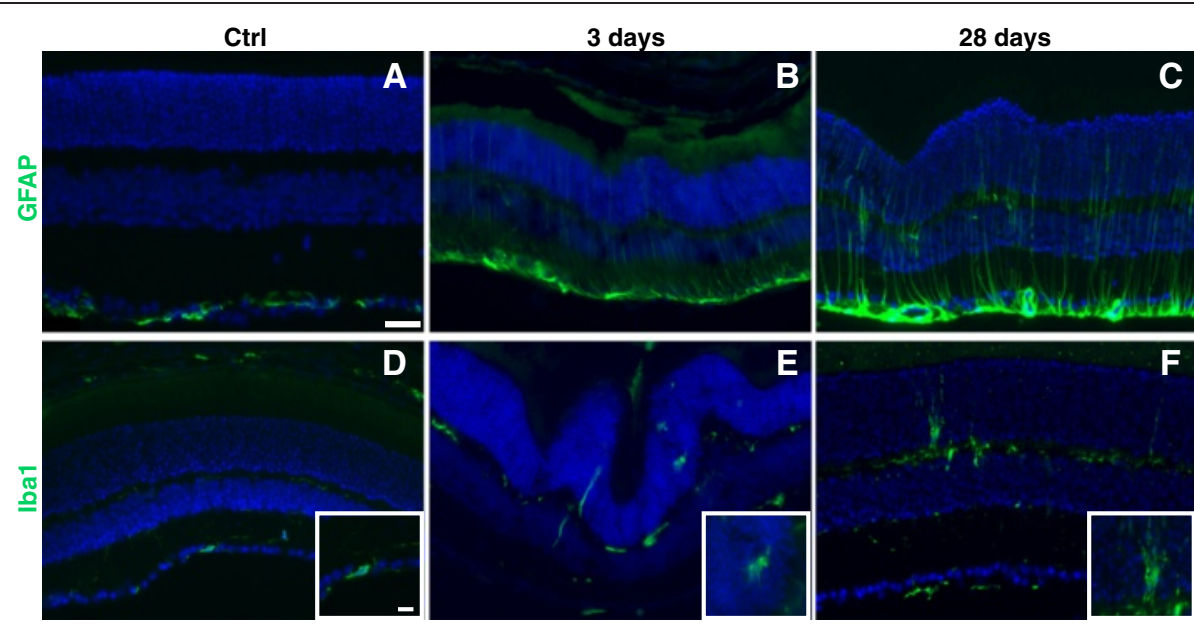

Figure 11 Both Müller glia and microglia become reactive after injury. (A-C) Representative epifluorescence micrographs of retinas immunolabeled for GFAP (green). (D-F) Representative epifluorescence micrographs of retinas immunolabeled for Iba-1 (green). Higher magnification images of representative microglia are present in inserts. The scale bar for the low magnification images is $25 \mu \mathrm{m}$ and applies to all images. The scale bar for the inserts is $10 \mu \mathrm{m}$ and applies to all images. DAPI is shown in blue.

seven days post-blast wave exposure, indicating a recovery in the outer retina. In contrast, the ERG $b_{\max }$ and spatial acuity threshold (visual acuity) continue to decrease at 28 days post-blast wave exposure. This suggests an ongoing dysfunction in the inner retina after blast wave exposure. These results are supported by the detection of a second wave of TUNEL-positive cells and significant optic nerve degeneration at 28 days post-blast wave exposure. The detection of TUNEL-positive cells prior to any degenerating axons in the optic nerve suggests that the axon degeneration is secondary to RGC death.

\section{Conclusions}

In summary, this study demonstrates that a blast overpressure airwave directed at the eyes of young DBA/

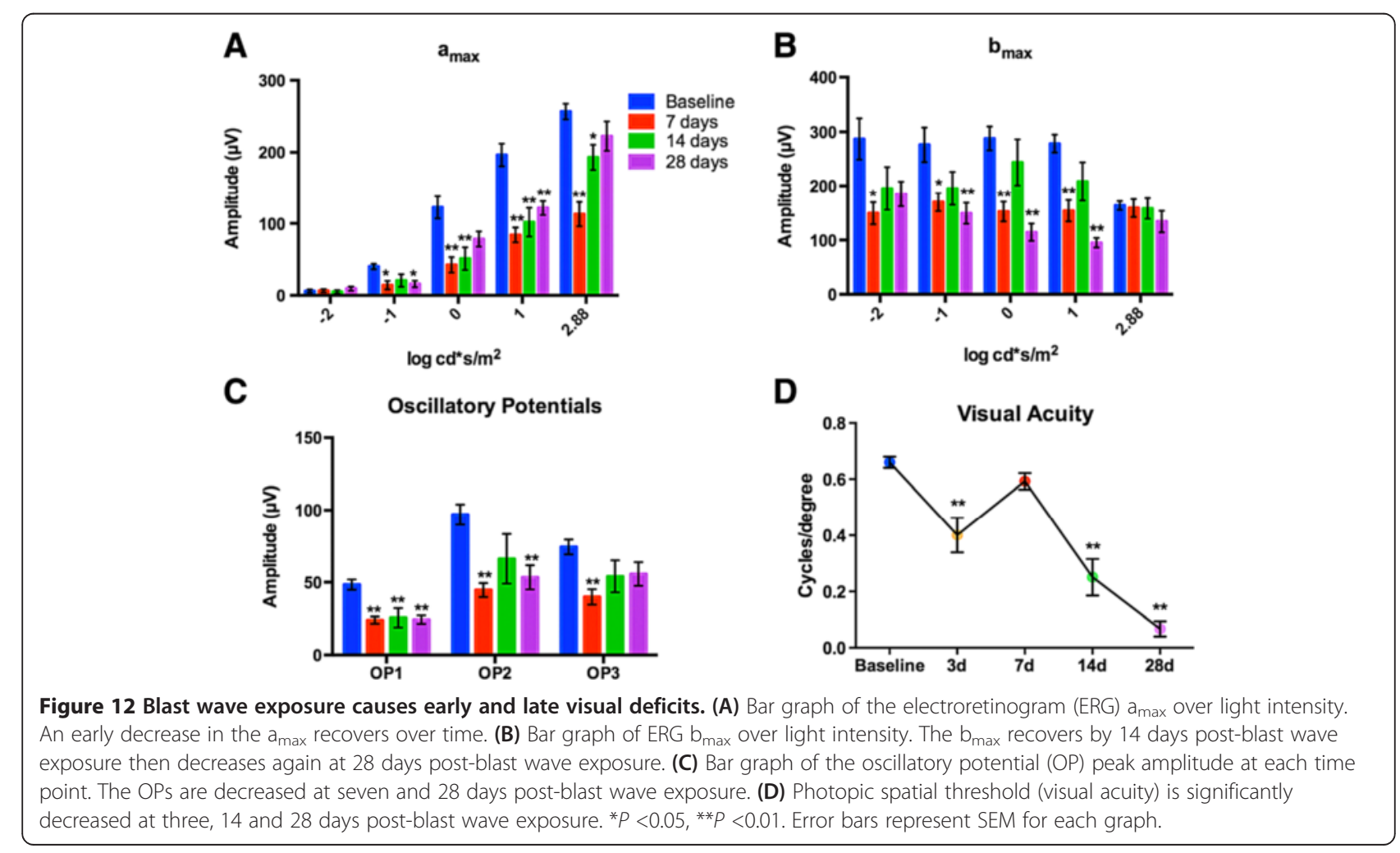


$2 \mathrm{~J}$ mice can be used as a model for open globe ocular trauma. This model is attractive because the globe remains intact, avoiding complications from potential bacterial infections. Treatment options for open globe trauma patients are very limited and none have shown a high degree of success. We expect that this model will be useful as a platform for identifying the mechanisms underlying ongoing vision loss and testing potential therapeutics for the treatment of open globe ocular trauma.

\section{Additional files}

Additional file 1: Figure S1. Treatment with non-medicated eye drops after blast reduces RPE damage. (A-B) Representative light micrographs of non-eye drop treated RPE (A) and eye drop treated RPE (B) at 3 days post-injury. The scale bar in (A) is $25 \mu \mathrm{m}$ and applies to both images. $\mathrm{RPE}=$ retinal pigment epithelium.

Additional file 2: Figure S2. Non-medicated eye drop treatment reduces immune infiltrate. (A-B) Representative light micrographs of cornea (A) and the ciliary body (B) from an eye drop treated eye at 3 days post-injury. The scale bar in (A) is $25 \mu \mathrm{m}$ and applies to both images.

Additional file 3: Figure S3. Nitrotyrosine immunolabeling is more focal after eye drop treatment. (A-B) Representative low and high magnification epifluorescence micrographs of a non-eye drop treated retina (A) and an eye drop treated retina (B) at 3 days post-injury. The scale bar in the low magnification image (A) is $250 \mu \mathrm{m}$ and applies to both low magnification images. The scale bar in the high magnification image (A) is $50 \mu \mathrm{m}$ and applies to both high magnification images, White boxes in the low magnification images correspond to the high magnification images.

Additional file 4: Figure S4. Microglial reactivity is limited after eye drop treatment. (A-B) Representative low and high magnification epifluorescence micrographs of a non-eye drop treated retina (A) and an eye drop treated retina (B) at 3 days post-injury. The white boxes denote reactive microglia and the arrows point to the corresponding high magnification images. The scale bar in the low magnification image (A) is $250 \mu \mathrm{m}$ and applies to both low magnification images. The scale bar in the high magnification image (A) is $50 \mu \mathrm{m}$ and applies to both high magnification images.

\section{Abbreviations \\ ACAID: Anterior chamber-associated immune deviation; DAPI: 4, 6-diamidino- 2-phenylindole; Endo: Endothelium; Epi: Epithelium; ERG: Electroretinogram; C3d: Complement 3d; CA: Corneal abrasions; CE: Corneal edema; \\ CNV: Corneal neovascularization; GCL: Ganglion cell layer; GFAP: Glial fibrillary acidic protein; IBA-1: lonized calcium binding adaptor molecule 1; INL: Inner nuclear layer; OCT: Optical coherence tomography; OKN: Optokinetic nystagmus; ONH: Optic nerve head; ONL: Outer nuclear layer; PBS: Phosphate buffered saline; PBT: Phosphate buffer with bovine serum albumin and triton $X$ 100; PFA: Paraformaldehyde; PMD: Pupilomotor deficit; \\ PVC: Polyvinylchloride; RGC: Retinal ganglion cell; RIP1: Receptor interacting protein 1; RIP3: Receptor interacting protein 3; RPE: Retinal pigment epithelium; TUNEL: TdT dUTP nick end labeling.}

\section{Competing interests}

The authors declare that they have no competing interests.

\section{Authors' contributions}

$\mathrm{CBA}, J H B$, and LD performed the laboratory experiments. CBA and TSR analyzed the data, interpreted the results and wrote the manuscript. All authors have contributed to, seen, and approved the final manuscript.

\section{Acknowledgements}

Grant support: Department of Defense W81XW-10-1-0528; NEl EY022349; Research to Prevent Blindness Career Development Award; Research to Prevent Blindness Unrestricted Funds (P Sternberg); P30-EY008126 (J Schall). DoD Non-Endorsement Disclaimer: The views, opinions and/or findings contained in this research paper are those of the authors and do not necessarily reflect the views of the Department of Defense and should not be construed as an official DoD/Army position, policy or decision unless so designated by other documentation. No official endorsement should be made.

\section{Author details}

'Vanderbilt Eye Institute, Vanderbilt University, 11425 MRB IV, 2213 Garland Ave., Nashville, TN 37232, USA. ${ }^{2}$ Vanderbilt Brain Institute, Vanderbilt University, 11425 MRB IV, 2213 Garland Ave., Nashville, TN 37232, USA. ${ }^{3}$ Department of Ophthalmology, University of Tennessee Health Science Center, 930 Madison Ave., Memphis, TN 38103, USA.

Received: 26 August 2014 Accepted: 1 November 2014

Published online: 29 November 2014

\section{References}

1. Hilber DJ: Eye injuries, active component, US Armed Forces, 2000-2010. Med Surveillance Mont Rep 2011, 18:2-7.

2. Frick K: Costs of military eye injury, vision impairment, and related blindness and vision dysfunction associated with traumatic brain injury (TBI) without eye injury. Nat Alliance Eye Vision Res 2012,1-16

3. May D, Kuhn F, Morris R, Witherspoon C, Danis R, Matthews G, Mann L: The epidemiology of serious eye injuries from the United States Eye Injury Registry. Graefe Arch Clin Exp Ophthalmol 2000, 238:153-157

4. McGwin G, Hall TA, Xie A, Owsley C: Trends in eye injury in the United States, 1992-2001. Invest Ophthalmol Vis Sci 2006, 47:521-527.

5. Phillips BN, Chun DW, Colyer M: Closed globe macular injuries after blasts in combat. Retina 2013, 33:371-379.

6. Weichel $\mathrm{E}$, Colyer M: Combat ocular trauma and systemic injury. Curr Opin Ophthalmol 2008, 19:519-525.

7. Scott R: The injured eye. Philos Trans R Soc B Biol Sci 2011, 366:251-260.

8. Hines-Beard J, Marchetta J, Gordon S, Chaum E, Geisert EE, Rex TS: A mouse model of ocular blast injury that induces closed globe anterior and posterior pole damage. Exp Eye Res 2012, 99:63-70.

9. Bricker-Anthony C, Hines-Beard J, Rex TS: Molecular changes and vision loss in a mouse model of closed-globe blast trauma. Invest Ophthalmol Vis Sci 2014, 55:4853-4862.

10. Mo J-S, Anderson MG, Gregory M, Smith RS, Savinova OV, Serreze DV Ksander BR, Streilein JW, John SW: By Altering ocular immune privilege, bone marrow-derived cells pathogenically contribute to DBA/2 J pigmentary glaucoma. J Exp Med 2003, 197:1335-1344

11. John S, Smith R, Savinova O, Hawes N, Chang B, Turnbull D, Davisson M, Roderick T, Heckenlively J: Essential iris atrophy, pigment dispersion, and glaucoma in DBA/2 J mice. Invest Ophthalmol Vis Sci 1998, 39:951-962.

12. Bosco A, Steele MR, Vetter ML: Early microglia activation in a mouse model of chronic glaucoma. J Comp Neurol 2011, 519:599-620.

13. Kraupp BG, Ruttkay-Nedecky B, Koudelka H, Bukowska K, Bursch W, Hermann RS: In situ detection of fragmented DNA (TUNEL assay) fails to discriminate among apoptosis, necrosis, and autolytic cell death: a cautionary Note. Hepatology 1995, 21:1465-1468.

14. Cockerham GC, Lemke S, Glynn-Milley C, Zumhagen L, Cockerham KP: Visual performance and the ocular surface in traumatic brain injury. Ocul Surf 2013, 11:25-34

15. Goldstein LE, Fisher AM, Tagge CA, Zhang X-L, Velisek L, Sullivan JA, Upreti C, Kracht JM, Ericsson M, Wojnarowicz MW: Chronic traumatic encephalopathy in blast-exposed military veterans and a blast neurotrauma mouse model. Sci Transl Med 2012, 4:134ra60-134ra60.

16. Mohan K, Kecova H, Hernandez-Merino E, Kardon RH, Harper MM: Retinal ganglion cell damage in an experimental rodent model of blast-mediated traumatic brain injury. Invest Ophthalmol Vis Sci 2013, 54:3440-3450. 
17. Zou YY, Kan EM, Lu J, Ng KC, Tan MH, Yao L, Ling EA: Primary blast injury-induced lesions in the retina of adult rats. J Neuroinflammation 2013, 10:79.

18. Prusky GT, Silver BD, Tschetter WW, Alam NM, Douglas RM:

Experience-dependent plasticity from eye opening enables lasting, visual cortex-dependent enhancement of motion vision. J Neurosci 2008, 28:9817-9827.

19. Alam M, Iabal M, Khan A, Khan SA: Ocular injuries in blast victims. JPMA J Pak Med Assoc 2012, 62:138.

doi:10.1186/s12974-014-0192-5

Cite this article as: Bricker-Anthony et al:: Exacerbation of blast-induced ocular trauma by an immune response. Journal of Neuroinflammation 2014 11:192.

\section{Submit your next manuscript to BioMed Central and take full advantage of:}

- Convenient online submission

- Thorough peer review

- No space constraints or color figure charges

- Immediate publication on acceptance

- Inclusion in PubMed, CAS, Scopus and Google Scholar

- Research which is freely available for redistribution 